\title{
Exploring Effects of Hydrocortisone on Implicit Motivation and Activity Inhibition: A Randomized Placebo-Controlled Study
}

\author{
Oliver C. Schultheiss ${ }^{1}$ - Uta S. Wiemers ${ }^{2}$. \\ Oliver T. Wolf ${ }^{2}$
}

Received: 16 September 2015 / Revised: 18 January 2016/Accepted: 21 January 2016 /

Published online: 1 February 2016

(C) Springer International Publishing 2016

\begin{abstract}
This study explored effects of experimental cortisol manipulation on changes in implicit motives. Thirty-six participants were randomly allocated to a hydrocortisone or a placebo condition, provided saliva samples that were assayed for cortisol, and wrote picture stories both before and after treatment. Stories were coded for motivational imagery related to power, achievement, and affiliation as well as for activity inhibition, a marker of functional brain lateralization during stress. Salivary cortisol was monitored before and after treatment. Hydrocortisone-treated participants, relative to controls, had lower post-treatment affiliation scores, but did not significantly differ from them on achievement, power, activity inhibition scores or number of words written. In the overall sample, increases in cortisol were associated with increases in achievement scores and decreases in activity inhibition. In the hydrocortisone condition, an increase in cortisol was associated with a decrease in power scores.
\end{abstract}

Keywords Randomized control trial $\cdot$ Motivation $\cdot$ Personality $\cdot$ Steroids · Glucocorticoids · Hypothalamic-pituitary-adrenal axis · Affiliation · Power ·

Achievement $\cdot$ Narrative fluency $\cdot$ Activity inhibition

This study was supported in part by the German Research Foundation (DFG) project B4 of Collaborative Research Center (SFB) 874 "Integration and Representation of Sensory Processes". We thank Sophie Albert and Andrea Schreiter for coding the stories.

\section{Oliver C. Schultheiss}

Oliver.Schultheiss@fau.de

1 Department of Psychology, Friedrich-Alexander University, Nägelsbachstrasse 49b, 91052 Erlangen, Germany

2 Department of Cognitive Psychology, Institute of Cognitive Neuroscience, Faculty of Psychology, Ruhr University Bochum, Bochum, Germany 


\section{Introduction}

Implicit motives, such as the needs for power (n Power), achievement (n Achievement), and affiliation (n Affiliation), represent nonconsciously operating affective preferences for specific classes of incentives (McClelland 1987; McClelland et al. 1989; Schultheiss 2008). They influence a wide range of motivational processes and behaviors (McClelland 1987; Schultheiss 2008) and do so, in part, by interacting with the endocrine system (McClelland 1989; Schultheiss 2013). A growing number of studies documents that the three above-mentioned implicit motives are all directly or indirectly associated with hypothalamic-pituitary-adrenal (HPA) axis functioning, predicting cortisol responses to a variety of motive-relevant situations (Schultheiss 2013; Schultheiss et al. 2014). What all previous studies on motive-hormone interactions have in common, though, is that such interactions were studied as correlations. Questions of causality have therefore remained elusive. In the present research, we address this issue for the first time by studying the effect of hydrocortisone administration, which represents a pharmacologically simulated strong HPA response, on changes in motivational needs.

$\mathrm{n}$ Affiliation is defined as a capacity to experience friendly, harmonious relationships as rewarding and social isolation as aversive (Schultheiss 2008). It has been linked to HPA axis function via its interaction with progesterone: Experimental arousal of affiliation motivation leads to a progesterone increase (Schultheiss et al. 2004; see also Maner et al. 2010) or to an association between increased $\mathrm{n}$ Affiliation and increased progesterone (Wirth and Schultheiss 2006). Wirth (2011) has argued that the $\mathrm{n}$ Affiliation-progesterone link serves to down-regulate HPA activation in response to stress, perhaps mediated by social contact seeking (cf. Maner et al. 2010; Taylor 2006), which leads to increased progesterone (Brown et al. 2009). This suggests an inverse functional relationship between $\mathrm{n}$ Affiliation and cortisol as a marker of HPA activation: the higher $\mathrm{n}$ Affiliation, the lower cortisol levels, but also the higher cortisol levels, the lower n Affiliation. A recent review by Sandi and Haller (2015) suggests that, consistent with the latter part of our hypothesis, pronounced stress leads to social withdrawal in humans and animals.

n Achievement represents a capacity to cherish the autonomous mastery of challenging tasks (Schultheiss and Brunstein 2005). Recent research suggests that while $\mathrm{n}$ Achievement may be positively associated with cortisol at rest or under conditions of mild challenge, it predicts strongly attenuated cortisol responses to competitive or social-evaluative challenges (Schultheiss et al. 2014; Yang et al. 2015), that is, situations that typically elicit robust HPA activation (e.g., Foley and Kirschbaum 2010). Schultheiss et al. (2014) have argued that this effect reflects the ability of achievement-motivated individuals to appraise difficult situations as challenges that can be mastered rather than as unmanageable threats (see also Schultheiss and Brunstein 2005). This leaves open the question of what happens if for some reason a person's HPA axis is strongly activated, resulting in a steep cortisol increase: does $n$ Achievement increase in a compensatory manner, too, with the goal of eventually putting the brakes on this response, or do elevated cortisol levels overwhelm and suppress n Achievement? We 
deem the former scenario more consistent with the mastery-oriented, emotion-regulating role of $\mathrm{n}$ Achievement than the latter.

$\mathrm{n}$ Power is defined as a capacity to derive pleasure from having an impact on others and to experience others' impact on oneself as aversive (Schultheiss 2008; Winter 1973). $n$ Power predicts HPA activation in response to social defeat (Wirth et al. 2006). Schultheiss (Schultheiss 2007; Stanton and Schultheiss 2009) has argued that a preponderance of HPA activation, relative to sympathetic nervous system (SNS) activation, leads to a reduction of the gonadal steroid testosterone and perhaps thereby also to a concomitant reduction of assertive motivation. Underscoring the latter point, Wiemers et al. (2015) have shown that a greater cortisol increase as a marker of HPA activation and a comparatively weaker increase in salivary alpha amylase as a marker of SNS activation predicted a reduction of $\mathrm{n}$ Power. This leads us to expect high cortisol to have an attenuating effect on $\mathrm{n}$ Power.

Activity inhibition has been identified as a recurrent moderator of the physiological and behavioral expression of implicit motives (Langens 2010; Schultheiss et al. 2009). It is based on the frequency of negations used in oral or written language. According to Schultheiss et al. (2009), greater levels of activity inhibition indicate a propensity towards stronger engagement of rightlateralized brain functions associated with attentional, emotional, and physiological processes, particularly during stress (for stress-associated effects of activity inhibition, see also Fontana et al. 1987; Langens and Stucke 2005; McClelland et al. 1985). No specific associations between activity inhibition and cortisol have been reported in the literature so far. But in light of the stress-associated effects of activity inhibition, we explored what effects, if any, a simulated HPA activation would have on this measure.

Because implicit motives and activity inhibition are assessed by scoring imaginative stories that research participants write in response to picture cues depicting a variety of social situations (see Schultheiss and Pang 2007), the frequency of such scores depend on the overall length of the stories. Thus, whatever effects of hydrocortisone administration on score changes are observed may merely reflect induced changes in narrative fluency. We therefore also examined the extent to which hydrocortisone treatment affected story word count.

We explored these issues in a double-blind, randomized, placebo-controlled study that was originally designed to study effects of hydrocortisone on memory (Wiemers and Wolf 2015). We assessed implicit motives, activity inhibition, and word count with a picture-story task administered before and after hydrocortisone or placebo administration. Immediately following the administration of hydrocortisone (i.e., exogenous cortisol) or placebo, participants completed the friendly Trier Social Stress Test (fTSST; Wiemers et al. 2013), a non-stressful social interaction with two individuals that provided sufficient time for hydrocortisone to become available in all body tissues, including the brain, and that was used to present a variety of everyday items for a later memory test (see Wiemers and Wolf 2015). Salivary levels of cortisol were monitored before and after hydrocortisone/placebo administration. The main focus of our analyses was on effects of hydrocortisone on implicit motives and related measures. But we also explored correlations of (changes in) cortisol and (changes in) implicit motive scores, activity inhibition, and word count. 


\section{Method}

\section{Participants}

Thirty-six participants ${ }^{1}$ (20 men) were included in this study (for admission criteria, see Wiemers and Wolf 2015). They had a mean age of 25.08 years $(\mathrm{SD}=2.72)$ and were paid $25 €$ upon study completion. Pregnant or menstruating women and women taking hormonal contraceptives were not admitted to the study. For female participants, an average of 19 days had elapsed since the last menstruation onset. The study was approved by the institutional review board of the Faculty of Medicine of the RuhrUniversity Bochum.

\section{Design}

The study had a double-blind, placebo-controlled, between-subjects design, with random allocation of participants to the hydrocortisone $(n=18 ; 10 \mathrm{men})$ or placebo ( $n=18 ; 10$ men) treatments of the experimental condition factor. Dependent variables were PSE scores for $\mathrm{n}$ Achievement, $\mathrm{n}$ Affiliation, $\mathrm{n}$ Power, activity inhibition, and word count assessed after the treatment (T2), controlling for baseline levels assessed before treatment $(\mathrm{T} 1)$.

\section{Procedure}

To control for circadian variations in cortisol, all participants were tested in the afternoon. After signing an informed consent form, participants completed a first picture-story task (T1) and provided a baseline saliva sample. At $30 \mathrm{~min}$, participants either received two pills totaling $20 \mathrm{mg}$ hydrocortisone (Jenapharm), a dosage that in previous studies was effective at eliciting memory effects similar to those observed under high stress (see Wolf 2009), or two placebo pills. They then completed the fTSST and at $50 \mathrm{~min}$ provided a second saliva sample. They were administered a second picture-story task (T2), provided a third saliva sample at $65 \mathrm{~min}$, worked on a task unrelated to the present research, and then provided a fourth saliva sample at $80 \mathrm{~min}$. After this, they were debriefed.

\footnotetext{
${ }^{1}$ Wiemers and Wolf (2015) excluded three participants from their analyses for the following reasons: One participant did not complete the second day of testing and thus skipped a critical aspect of the memory measure this study focused on but that is irrelevant for the data we report here; one participant had once participated in a regular Trier Social Stress Test (but not the fTSST) in another lab, which was a threat to the internal validity of the memory part of the Wiemers and Wolf (2015) paper, but not for our analyses; and one participant in the placebo group showed a slightly stronger cortisol response than the rest of the placebo group. However, because the placebo group showed little cortisol variations in absolute terms and the aberrant data point was only $2.5 \mathrm{SD}$ units above the placebo group, not the $3 \mathrm{SD}$ units more typically considered to be a stringent threshold for the definition of an outlier, this did not pose a threat to the validity of the present findings, either. We therefore retained the three participants excluded in the analyses reported by Wiemers and Wolf (2015).
} 


\section{Materials}

Friendly TSST The fTSST was included as a social interaction task and presented material for an incidental memory task conducted on the next day (see Wiemers and Wolf 2015, for findings). In other research, it is typically used as a non-stressful control condition for the TSST. Wiemers et al. (2015) have shown that it is motivationally neutral in the sense of not eliciting significant changes in implicit motives or activity inhibition. After a 5-min preparation time during which participants made notes about their school and university experiences, career aspirations, hobbies, and favorite book or movie, they gave a 10-min free speech about their life and career aspirations in front of a committee consisting of a man and a woman. The committee reacted in a friendly manner by nodding and smiling, thus providing participants with a feeling of safety and appreciation. The friendly and non-threatening nature of the fTSST was documented in this and other studies by task-induced increases in positive affect and no increases in negative affect or salivary cortisol (see Wiemers and Wolf 2015).

Motive Assessment Participants' implicit motives were assessed by a 6-picture Picture Story Exercise (PSE; Schultheiss and Pang 2007). Pictures were divided into two sets of three pictures each, with Set A consisting of the pictures Boxer, Women in Laboratory, and Trapeze Artists, and Set B consisting of the pictures Soccer Duel, Gymnast, and Workers (see Schultheiss and Pang 2007, for details). Pictures were selected for their ability to elicit high levels, and thus high variance, of power and achievement imagery; they also yield, at a lower level, scores for $\mathrm{n}$ Affiliation (Pang 2010; Schultheiss and Pang 2007). One set was presented before hydrocortisone or placebo administration (T1), the other 20 min after (T2). Set sequence (AB, BA) was counterbalanced across participants and orthogonal to experimental conditions. Within each set, presentation of pictures was random. Pictures were presented on a computer screen and participants wrote their stories in a text box on the computer. Two coders who were blind to participants' experimental condition later independently scored all stories for imagery related to $\mathrm{n}$ Power (e.g, controlling, impressing, persuading others), n Achievement (e.g., unique accomplishments or doing a task well), and n Affiliation (e.g., dialog or expression of friendly feelings towards others) using Winter's (1991, 1994) Manual for Scoring Motive Imagery in Running Text. The coders had previously exceeded $85 \%$ inter-scorer agreement on German calibration materials prescored by experts and were blind to experimental condition. Their interrater reliability (Lin's 1989, concordance correlation coefficient) for $\mathrm{n}$ Power, $\mathrm{n}$ Achievement, and $\mathrm{n}$ Affiliation was, respectively, .91, .69, and .87 at T1 and .88, .91, and .96 at T2. Motive scores were averaged across coders for all subsequent analyses. Story word count and activity inhibition, defined as the frequency of the German negation nicht (see McClelland 1979), were determined with the help of a word processor. Because motive and activity inhibition score distributions were skewed, we subjected them to a square-root transformation after adding a constant of 1 .

Hormone Assessment Participants were asked to refrain from eating and drinking anything except water $1 \mathrm{~h}$ before testing. Saliva was collected using Salivettes (Sarstedt, Nuernbrecht, Germany) and cortisol was measured with an immunoassay (IBL, Hamburg, Germany). Inter- and intra-assay coefficients of variation were below 
$10 \%$. Because salivary cortisol had a skewed distribution at all four assessments, we subjected values to a log-transformation after adding a constant of 1 and used these transformed values for all inferential statistics. The three post-fTSST cortisol measurements were averaged to derive an overall measure of hydrocortisone- or placeboinduced cortisol changes. For the hydrocortisone and for the placebo group, untransformed baseline (T1) cortisol levels were $10.45 \mathrm{nmol} / \mathrm{l}(S D=6.20)$ and $10.29 \mathrm{nmol} / \mathrm{l}$ $(S D=5.81)$ and untransformed averaged post-fTSST (T2) cortisol levels were $295.04 \mathrm{nmol} / \mathrm{l}(S D=250.87)$ and $11.41 \mathrm{nmol} / 1(S D=6.73)$, respectively.

Statistical Analyses Due to the exploratory nature of this study, we provide full descriptive data on the PSE measures along with significance test and effect size measures (d) for between- and within-group differences. To test effects of experimental condition on T2 PSE measures, we used an ANCOVA-type regression approach, which is more powerful than repeated-measures ANOVA in randomized designs (see Van Breukelen 2006), in which PSE scores at T1 and PSE sequence (AB, BA) were held constant. To test concurrent associations between PSE measures and cortisol, we used Pearson correlations and partial correlations (controlling for experimental condition). To test associations between changes in PSE measures and changes in cortisol, we calculated bipartial correlation coefficients for T2 measures, with their respective T1 levels held constant (Cohen and Cohen 1983).

\section{Results}

\section{PSE Scores}

Table 1 and Fig. 1 display the results for motive and activity inhibition scores as well as overall word count. Hydrocortisone participants did not differ from placebo controls in their T1 n Affiliation levels, but had significantly lower levels at T2. This difference was due to a non-significant reduction in $\mathrm{n}$ Affiliation due to hydrocortisone treatment, whereas the placebo group did not change in their $\mathrm{n}$ Affiliation levels from T1 to T2 at all. As shown in Table 2, regression analysis indicated a trend-level $(p=.08)$ negative effect of experimental condition on $\mathrm{n}$ Affiliation at T2 with motive scores at T1 and PSE sequence covaried out. ${ }^{2}$

Hydrocortisone participants had higher mean $n$ Achievement scores at $\mathrm{T} 2$ than at T1 or than placebo-group participants at T2. However, these differences did not reach significance. Likewise, the regression analysis reported in Table 2 indicates a positive, but non-significant effect of hydrocortisone treatment on T2 n Achievement.

$\mathrm{n}$ Power scores did not substantially differ within or between groups for T1 and T2. Regression analysis confirmed this with a near-null effect of experimental condition on T2 n Power.

\footnotetext{
${ }^{2}$ When we ran a repeated-measures ANOVA with simultaneous testing of effects of experimental condition, PSE sequence, and their interaction, the specific effect of experimental condition was significant, $F(1,32)=4.43, p=.04$.
} 
Table 1 Mean (SD) values for PSE raw motive, AI, and word count scores and significance tests and effect sizes for within- and between-group differences

\begin{tabular}{|c|c|c|c|c|c|}
\hline & $\mathrm{T} 1$ & $\mathrm{~T} 2$ & $t$ & $d$ & $p$ \\
\hline \multicolumn{6}{|l|}{$n$ Affiliation $^{a}$} \\
\hline Hydrocortisone & $1.33(1.64)$ & $0.67(0.86)$ & -1.62 & -0.48 & .12 \\
\hline Placebo & $2.06(2.41)$ & $2.14(2.61)$ & 0.10 & 0.03 & .92 \\
\hline$t$ & -0.94 & -2.26 & & & \\
\hline$d$ & -0.31 & 0.55 & & & \\
\hline$p$ & .35 & .03 & & & \\
\hline \multicolumn{6}{|l|}{$n$ Achievement ${ }^{a}$} \\
\hline Hydrocortisone & $3.75(1.23)$ & $4.83(3.31)$ & 1.02 & 0.34 & .32 \\
\hline Placebo & $4.11(2.04)$ & 3.58 (1.64) & -0.76 & -0.25 & .46 \\
\hline$t$ & -0.42 & 1.25 & & & \\
\hline$d$ & -0.14 & 0.42 & & & \\
\hline$p$ & .68 & .22 & & & \\
\hline \multicolumn{6}{|l|}{$n$ Power $^{a}$} \\
\hline Hydrocortisone & $3.28(1.57)$ & $3.47(1.60)$ & 0.39 & 0.10 & .70 \\
\hline Placebo & $3.11(2.02)$ & $3.47(2.93)$ & 0.35 & 0.08 & .72 \\
\hline$t$ & 0.44 & 0.31 & & & \\
\hline$d$ & 0.15 & 0.10 & & & \\
\hline$p$ & .66 & .76 & & & \\
\hline \multicolumn{6}{|l|}{ Activity inhibition ${ }^{a}$} \\
\hline Hydrocortisone & $1.67(1.81)$ & $1.78(1.31)$ & 0.58 & 0.15 & .57 \\
\hline Placebo & $2.56(1.82)$ & $2.61(1.72)$ & 0.13 & 0.03 & .90 \\
\hline$t$ & -1.65 & -1.53 & & & \\
\hline$d$ & -0.55 & -0.51 & & & \\
\hline$p$ & .11 & .14 & & & \\
\hline \multicolumn{6}{|l|}{ Word count } \\
\hline Hydrocortisone & $279(91)$ & $272(73)$ & -0.53 & -0.09 & .60 \\
\hline Placebo & $303(78)$ & 307 (79) & 0.51 & 0.06 & .62 \\
\hline$t$ & -0.83 & -1.41 & & & \\
\hline$d$ & -0.28 & -0.47 & & & \\
\hline$p$ & .41 & .17 & & & \\
\hline
\end{tabular}

${ }^{a} t$-tests and effect size estimates are based on square-root-transformed variables

Activity inhibition was slightly higher in the placebo group than in the hydrocortisone group both at T1 and T2 (for the main effect of experimental condition, $\mathrm{F}[1,33]=4.04$, partial $\left.\eta^{2}=.109, p=.05\right)$. Regression analysis suggested that after holding T1 levels and PSE sequence constant, experimental condition had a negative effect on $\mathrm{T} 2$ activity inhibition, but the effect failed to become significant.

Word count did not substantially differ within or between groups for T1 and T2. Regression analysis suggested a negative, but non-significant effect of 


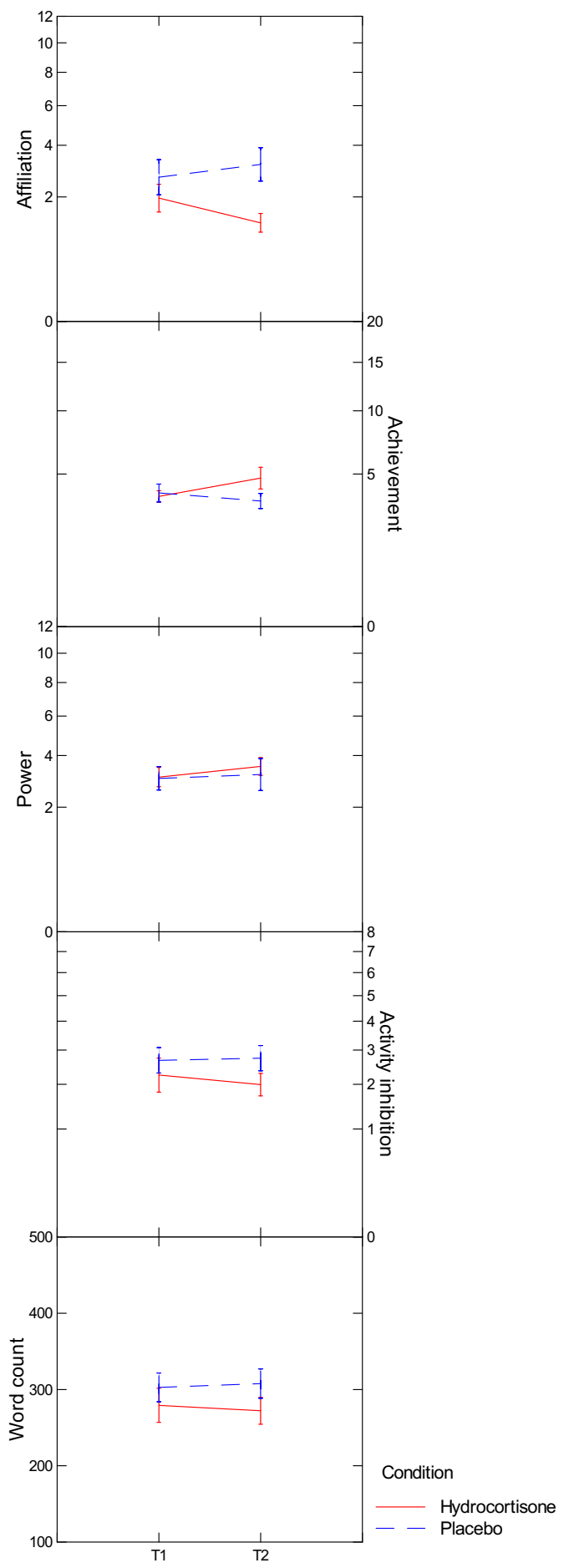

Fig. 1 Effects of experimental condition and assessment (T1, T2) on PSE motive, activity inhibition, and word count scores. Error bars represent SEM 
Table 2 Unstandardized regression coefficients (SE) from simultaneous regressions of T2 PSE scores on T1 PSE scores, PSE sequence (AB, BA), and experimental condition $(0=$ placebo, $1=$ hydrocortisone $)$

\begin{tabular}{llllll}
\hline & $\mathrm{n} \mathrm{Affiliation}^{a}$ & $\mathrm{n} \mathrm{Achievement}^{a}$ & $\mathrm{n} \mathrm{Power}^{a}$ & Activity inhibition $^{a}$ & Word count \\
\hline $\mathrm{T} 1$ & $0.480(0.150)^{* * *}$ & $0.251(0.218)$ & $0.581(0.205)^{* *}$ & $0.335(0.141)^{*}$ & $0.678(0.096)^{* * *}$ \\
PSE Sequence & $-0.433(0.167)^{*}$ & $0.557(0.167)^{* * *}$ & $0.089(0.180)$ & $0.133(0.139)$ & $22.18(15.87)$ \\
Condition & $-0.282(0.155)^{+}$ & $0.207(0.159)$ & $0.015(0.165)$ & $-0.138(0.142)$ & $-20.98(15.06)$ \\
\hline
\end{tabular}

${ }^{a} \mathrm{~T} 1$ and $\mathrm{T} 2$ scores represent square-root-transformed variables

$+p<.10, * p<.05, * * p<.01, * * * p<.005$

experimental condition on word count. None of these effects was significantly moderated by gender.

\section{Associations Between Cortisol and PSE Scores}

These analyses focused on correlations between variations in cortisol (changes) and variations in PSE score (changes) above and beyond the main effects of treatment reported above by either partialing for effects of treatment (which were massive in the case of cortisol, see Wiemers and Wolf 2015) or focusing on correlations of changes within each experimental condition (see Table 3).

Examination of associations between $\mathrm{n}$ Affiliation and cortisol at $\mathrm{T} 1$ and $\mathrm{T} 2$ as well as between changes in $\mathrm{n}$ Affiliation and changes in cortisol from $\mathrm{T} 1$ to T2 in the overall sample or within each condition suggested that cortisol increases were non-significantly correlated with decreases in $\mathrm{n}$ Affiliation, an effect that was consistent with the reported negative effect of hydrocortisone treatment on $n$ Affiliation reported above.

Higher $\mathrm{n}$ Achievement was correlated with lower cortisol at $\mathrm{T} 1$, but higher cortisol at T2, resulting in a marginally significant positive correlation between $\mathrm{n}$ Achievement changes and cortisol changes. This effect was slightly stronger in the hydrocortisone than in the placebo condition, but the difference was not significant.

Results for $\mathrm{n}$ Power show the opposite pattern: while higher $\mathrm{n}$ Power was correlated with higher cortisol at T1, it was correlated with lower cortisol at T2. As a consequence, cortisol increases from $\mathrm{T} 1$ to $\mathrm{T} 2$ were associated with $\mathrm{n}$ Power decreases in the overall sample. This association was strong and significant in the hydrocortisone condition but absent in the placebo condition with its much smaller and unsystematic cortisol changes. The difference between the correlation coefficients did not quite reach the .10 threshold, though.

At both T1 and T2, higher cortisol was associated with lower activity inhibition, although the effect was significant at T2 only. In the overall sample, cortisol increases were correlated with activity inhibition decreases. This effect was very similar in the hydrocortisone and placebo conditions, suggesting that the absolute amount of cortisol change was less important for activity inhibition changes than the direction of the change.

For PSE word count, concurrent and change correlations with cortisol were weak and unsystematic, suggesting that writing fluency was largely independent of cortisol levels. 


\section{Discussion}

In this study, we explored for the first time effects of hydrocortisone administration on changes in measures of implicit motivation and activity inhibition. Our results suggest that hydrocortisone administration, mimicking a strong hormonal stress response, has a damping effect on $\mathrm{n}$ Affiliation, the need to have friendly, harmonious relationships, as indicated by a significant post-treatment $\mathrm{n}$ Affiliation difference between experimental groups and a marginal main effect of experimental condition on residual $n$ Affiliation changes (this effect does not appear to be due to the fTSST, as this task by itself had no detectable impact on $\mathrm{n}$ Affiliation in a previous study by Wiemers et al. 2015). Analysis of correlation patterns, although not significant, corroborate this conclusion by indicating that greater cortisol increases tended to be associated with greater reductions in $\mathrm{n}$ Affiliation. These findings are consistent with the general observation that pronounced stress leads to reduced sociality (Sandi and Haller 2015) and more specifically the notion of an inverse relationship between $n$ Affiliation and HPA axis activation (Wirth 2011).

Although missing the significance threshold, findings for hydrocortisone effects on $n$ Achievement were somewhat suggestive of a compensatory increase of this motivational need in verum-treated participants. Correlation patterns similarly indicated that a cortisol increase was associated at a marginal significance level with an $\mathrm{n}$ Achievement increase in the overall sample. If taken at face value, these observations suggest that hormonal changes signifying a massive HPA axis activation elicit, or are accompanied by, the motivation to autonomously master a challenging situation, a response that may result in adaptive cognitions and behaviors aimed at downregulating HPA axis activation.

Hydrocortisone administration had no discernible direct effect on $\mathrm{n}$ Power, although greater cortisol increases in the verum group, but not in the placebo group, were associated with steeper reductions in this motivational need. We explain this apparently contradictory set of findings as follows: Low to moderate HPA axis activation, particularly if accompanied by stronger SNS activation (see Wiemers and Wolf 2015, for salivary alpha amylase findings for this study), leads to an increase in $\mathrm{n}$ Power, as observed previously by Wiemers et al. (2015) and represented by hydrocortisone-

Table 3 Pearson correlations between concurrently assessed PSE measures and log-transformed cortisol at T1 (zero-order correlation) and T2 (partialled for experimental condition), bipartial correlations between PSE scores at T2 (with T1 levels held constant) and log-transformed averaged cortisol at T2 (with log-transformed cortisol at T1 held constant) for the overall sample (partialled for experimental condition) and experimental conditions, and tests of correlation differences between hydrocortisone- and placebo-group coefficients

\begin{tabular}{lllllll}
\hline & T1 & T2 & $\begin{array}{l}\text { Overall } \\
\text { change }\end{array}$ & $\begin{array}{l}\text { Hydrocortisone } \\
\text { change }\end{array}$ & $\begin{array}{l}\text { Placebo } \\
\text { change }\end{array}$ & Difference \\
\hline n Affiliation $^{a}$ & -.01 & -.17 & -.19 & -.14 & -.36 & $\mathrm{Z}=0.65, p=52$ \\
n Achievement $^{a}$ & $-.29+$ & .27 & $.29+$ & .32 & .20 & $\mathrm{Z}=-0.35, p=.73$ \\
n Power $^{a}$ & .19 & -.28 & -.26 & $-.51^{*}$ & .02 & $\mathrm{Z}=-1.60, p=.11$ \\
Activity inhibition $^{a}$ & -.17 & $-.38^{*}$ & $-.36^{*}$ & $-.41+$ & -.40 & $\mathrm{Z}=-0.03, p=.98$ \\
Word count & -.17 & -.10 & .07 & -.02 & .30 & $\mathrm{Z}=0.90, p=.37$ \\
\hline
\end{tabular}

${ }^{a} \mathrm{~T} 1$ and $\mathrm{T} 2$ scores represent square-root-transformed variables

$+p<.10, * p<.05$ 
treated participants with comparatively lower cortisol increases. Strong HPA axis activation, particularly if it outstrips SNS activation, on the other hand, is hypothesized to lead to reduced n Power (cf. Schultheiss 2007). In the hydrocortisone group, both scenarios happened. Some participants had a relatively moderate cortisol increase, leading to $\mathrm{n}$ Power increases, whereas other participants had medium to strong cortisol increases, leading to a reduction of $n$ Power. At the level of testing a main effect of hydrocortisone on $\mathrm{n}$ Power, the effects cancelled each other out, but it was apparent at the level of correlational analyses.

Similarly, although hydrocortisone administration had no effect on activity inhibition, cortisol increases were significantly associated with decreases in this variable. This suggests a cortisol-associated shift from right- to left-hemispheric processing, an effect that is broadly consistent with other reports of shifts in laterality resulting from stress (e.g., Gerhards et al. 1997) or, more directly, from hydrocortisone administration (e.g., Tops et al. 2006). For the cortisol/activity inhibition association we observed no difference between the verum and the placebo groups, which suggests that the absolute magnitude of cortisol change, which was vastly different between the two groups, was less important than its direction.

Experimental condition failed to have any impact on narrative fluency on the PSE and word count was not substantially associated with cortisol (changes) at any time or in any group. Hence, variations in this variable cannot account for the previously discussed implicit motive and activity inhibition findings. Neither did we find any evidence that effects of experimental condition on any of these variables depended on participant gender.

The overall picture emerging from the motive change analysis suggests that strong HPA activation, as simulated in this study by hydrocortisone administration, leads to a reduction of social motivation, with an overall negative effect in the case of $\mathrm{n}$ Affiliation and one for medium or high cortisol increases in the case of $\mathrm{n}$ Power. At the same time, rising cortisol appears to be associated with an increase in $\mathrm{n}$ Achievement, a motivational disposition that focuses on mastering the non-social environment and has been implicated in the regulation of emotional states, including stress (Schultheiss and Brunstein 2005).

\section{Limitations}

Despite its strengths as a randomized control trial, our study has a number of limitations that need to be considered when evaluating the validity of our findings. First off, our findings are based on a small sample, which is typical for research involving the administration of pharmacological agents, but nevertheless poses limitations in terms of statistical power. Given our sample size, we had a power of $83 \%$ to reliably detect standardized mean differences of 1 , that is, a large effect size. For the largest mean difference we observed in this study (between-group difference for n Affiliation at T2), our power was only $36 \%$. Because of this, and also because we did not correct for multiple tests in our analyses, findings obtained in the present study should be viewed as strictly exploratory before they are replicated in future studies with sufficiently large samples.

Second, the hydrocortisone dosage used in the present research resulted in cortisol increases in saliva (and hence also in blood) that were clearly supraphysiological. This 
certainly ensured saturation of glucocorticoid receptor binding, plus additional binding to mineralocorticoid receptors. But given the well-known nonlinear does-response relationships between cortisol and its physiological and psychological target functions, it remains an open question whether we would have seen similar effects as those reported when using doses mimicking the maximum physiological response the HPA axis is capable of. Likewise, the present research did not address to what extent the observed effects were due not only to verum-induced cortisol increases themselves, but also to the subsequent inhibition of other components of the HPA axis (e.g., reductions in corticotropin-releasing hormone and adrenocorticotropic hormone).

A third, related issue concerns the fact that hydrocortisone administration mimicked an extreme HPA response in the absence of any identifiable psychological or physiological stressor. It is therefore unclear if we would have obtained the same results had we paired hydrocortisone administration with situations suitable as motivational stressors (e.g., social defeat in the case of $n$ Power, social rejection in the case of $n$ Affiliation, etc). In a related vein, despite the fact that the fTSST represented a nonstressful social interaction with no discernible effects on implicit motives in previous research (Wiemers et al. 2015), this aspect of our study was not varied by, for instance, contrasting it with a no-interaction waiting condition. It therefore remains to be determined whether the use of this task interacted with hydrocortisone administration in some way to influence motivational changes.

Finally, although interrater reliabilities for motive scoring were generally good, the one exception to this rule was the less than satisfactory reliability for $\mathrm{n}$ Achievement at T1. Our failure to document a significant effect of hydrocortisone on $\mathrm{n}$ Achievement at $\mathrm{T} 2$ may thus be due in part to the lack of a reliable comparison measure (or covariate in the case on regression analyses) at $\mathrm{T} 1$.

\section{Conclusion}

To sum up, the present exploratory study represents the first examination of hormone administration effects on changes in implicit motivation and activity inhibition. We found evidence for an attenuation of $\mathrm{n}$ Affiliation due to experimentally increased cortisol and associations between cortisol changes and $n$ Achievement (positive) and $\mathrm{n}$ Power and activity inhibition (negative). These results suggest that experimental hormone manipulations can alter motivational states and that hormone administration may therefore be a viable route for further research on implicit motivation in humans.

\section{References}

Brown, S. L., Fredrickson, B. L., Wirth, M. M., Poulin, M. J., Meier, E. A., Heaphy, E. D., \& Schultheiss, O. C. (2009). Social closeness increases salivary progesterone in humans. Hormones and Behavior, 56, 108-111.

Cohen, J., \& Cohen, P. (1983). Applied multiple regression/correlation analysis for the behavioral sciences (2 ed., ). Hillsdale NJ: Erlbaum.

Foley, P., \& Kirschbaum, C. (2010). Human hypothalamus-pituitary-adrenal axis responses to acute psychosocial stress in laboratory settings. Neuroscience and Biobehavioral Reviews, 35(1), 91-96. doi:10.1016/j. neubiorev.2010.01.010. 
Fontana, A. F., Rosenberg, R. L., Marcus, J. L., \& Kerns, R. D. (1987). Type a behavior pattern, inhibited power motivation, and activity inhibition. Journal of Personality and Social Psychology, 52, 177-183.

Gerhards, F., Yehuda, R., Shoham, M., \& Hellhammer, D. H. (1997). Abnormal cerebral laterality in posttraumatic stress disorder. Annals of the New York Academy of Sciences, 821, 482-485.

Langens, T. A. (2010). Activity inhibition. In O. C. Schultheiss, \& J. C. Brunstein (Eds.), Implicit motives (pp. 89-115). New York, NY: Oxford University Press.

Langens, T. A., \& Stucke, T. S. (2005). Stress and mood: the moderating role of activity inhibition. Journal of Personality, 73(1), 47-78.

Lin, L. I. (1989). A concordance correlation coefficient to evaluate reproducibility. Biometrics, 45(1), 255-268.

Maner, J. K., Miller, S. L., Schmidt, N. B., \& Eckel, L. A. (2010). The endocrinology of exclusion: rejection elicits motivationally tuned changes in progesterone. Psychological Science, 21(4), 581-588.

McClelland, D. C. (1979). Inhibited power motivation and high blood pressure in men. Journal of Abnormal Psychology, 88, 182-190.

McClelland, D. C. (1987). Human motivation. New York: Cambridge University Press.

McClelland, D. C. (1989). Motivational factors in health and disease. American Psychologist, 44, 675-683.

McClelland, D. C., Ross, G., \& Patel, V. (1985). The effect of an academic examination on salivary norepinephrine and immunoglobulin levels. Journal of Human Stress, 11, 52-59.

McClelland, D. C., Koestner, R., \& Weinberger, J. (1989). How do self-attributed and implicit motives differ? Psychological Review, 96, 690-702.

Pang, J. S. (2010). Content coding methods in implicit motive assessment: standards of measurement and best practices for the picture story exercise. In O. C. Schultheiss, \& J. C. Brunstein (Eds.), Implicit motives (pp. 119-150). New York, NY: Oxford University Press.

Sandi, C., \& Haller, J. (2015). Stress and the social brain: behavioural effects and neurobiological mechanisms. Nature Reviews Neuroscience, 16(5), 290-304. doi:10.1038/nrn3918.

Schultheiss, O. C. (2007). A biobehavioral model of implicit power motivation arousal, reward and frustration. In E. Harmon-Jones, \& P. Winkielman (Eds.), Social neuroscience: integrating biological and psychological explanations of social behavior (pp. 176-196). New York: Guilford.

Schultheiss, O. C. (2008). Implicit motives. In O. P. John, R. W. Robins, \& L. A. Pervin (Eds.), Handbook of personality: theory and research (3 ed., pp. 603-633). New York: Guilford.

Schultheiss, O. C. (2013). The hormonal correlates of implicit motives. Social and Personality Psychology Compass, 7(1), 52-65. doi:10.1111/spc3.12008.

Schultheiss, O. C., \& Brunstein, J. C. (2005). An implicit motive perspective on competence. In A. J. Elliot, \& C. Dweck (Eds.), Handbook of competence and motivation (pp. 31-51). New York: Guilford.

Schultheiss, O. C., \& Pang, J. S. (2007). Measuring implicit motives. In R. W. Robins, R. C. Fraley, \& R. Krueger (Eds.), Handbook of research methods in personality psychology (pp. 322-344). New York: Guilford.

Schultheiss, O. C., Wirth, M. M., \& Stanton, S. J. (2004). Effects of affiliation and power motivation arousal on salivary progesterone and testosterone. Hormones and Behavior, 46(5), 592-599.

Schultheiss, O. C., Riebel, K., \& Jones, N. M. (2009). Activity inhibition: a predictor of lateralized brain function during stress? Neuropsychology, 23, 392-404.

Schultheiss, O. C., Wiemers, U., \& Wolf, O. T. (2014). Implicit need for achievement predicts attenuated cortisol responses to difficult tasks. Journal of Research in Personality, 48, 84-92. doi:10.1016/j.jrp.2013.10.004.

Stanton, S. J., \& Schultheiss, O. C. (2009). The hormonal correlates of implicit power motivation. Journal of Research in Personality, 43, 942-949.

Taylor, S. E. (2006). Tend and befriend: biobehavioral bases of affiliation under stress. Current Directions in Psychological Science, 15, 273-277.

Tops, M., Wijers, A. A., Koch, T., \& Korf, J. (2006). Modulation of rotational behavior in healthy volunteers by cortisol administration. Biological Psychology, 71(3), 240-243. doi:10.1016/j.biopsycho.2005.06.007.

Van Breukelen, G. J. (2006). ANCOVA versus change from baseline: more power in randomized studies, more bias in nonrandomized studies [corrected]. Journal of Clinical Epidemiology, 59(9), 920-925. doi:10. 1016/j.jclinepi.2006.02.007.

Wiemers, U.S., \& Wolf, O.T. (2015). Cortisol broadens memory of a non-stressful social interaction. Psychopharmacology. 232(10), 1727-1733. doi:10.1007/s00213-014-3808-7.

Wiemers, U. S., Schoofs, D., \& Wolf, O. T. (2013). A friendly version of the trier social stress test does not activate the HPA axis in healthy men and women. Stress, 16(2), 254-260. doi:10.3109/ 10253890.2012 .714427$.

Wiemers, U. S., Schultheiss, O. C., \& Wolf, O. T. (2015). Public speaking in front of an unreceptive audience increases implicit power motivation and its endocrine arousal signature. Hormones and Behavior, 71, 6974. doi:10.1016/j.yhbeh.2015.04.007. 
Winter, D. G. (1973). The power motive. New York: Free Press.

Winter, D. G. (1991). Measuring personality at a distance: development of an integrated system for scoring motives in running text. In D. J. Ozer, J. M. Healy, \& A. J. Stewart (Eds.), Perspectives in personality (vol. 3, pp. 59-89). London: Jessica Kingsley.

Winter, D. G. (1994). Manual for scoring motive imagery in running text (4 ed., ). Department of Psychology, University of Michigan, Ann Arbor: Unpublished manuscript.

Wirth, M. M. (2011). Beyond the HPA axis: progesterone-derived neuroactive steroids in human stress and emotion. Frontiers in Endocrinology., 2, 1-14. doi:10.3389/fendo.2011.00019.

Wirth, M. M., \& Schultheiss, O. C. (2006). Effects of affiliation arousal (hope of closeness) and affiliation stress (fear of rejection) on progesterone and cortisol. Hormones and Behavior, 50, 786-795.

Wirth, M. M., Welsh, K. M., \& Schultheiss, O. C. (2006). Salivary cortisol changes in humans after winning or losing a dominance contest depend on implicit power motivation. Hormones and Behavior, 49(3), $346-352$.

Wolf, O. T. (2009). Stress and memory in humans: twelve years of progress? Brain Research, 1293 142-154. doi:10.1016/j.brainres.2009.04.013

Yang, F., Ramsay, J. E., Schultheiss, O. C., \& Pang, J. S. (2015). Need for achievement moderates the effect of motive-relevant challenge on salivary cortisol changes. Motivation and Emotion, 39(3), 321-334. doi:10. 1007/s11031-014-9465-7. 